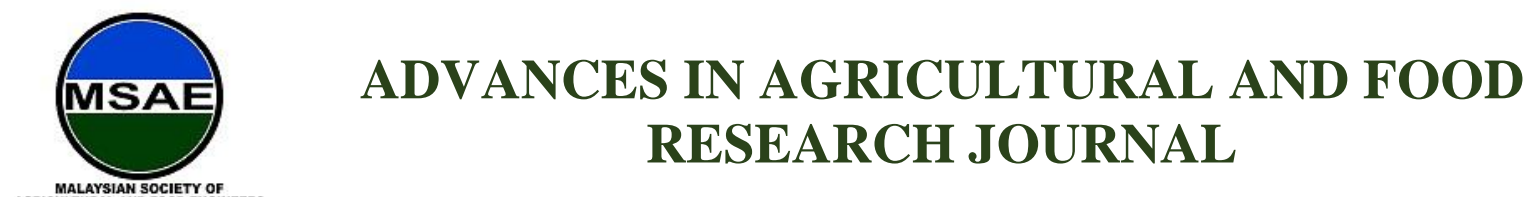

HH PUBLISHER

Original Research Article

\title{
Isolation of nanocellulose from Saba' (Musa acuminata $x$ balbisiana) banana peel by one-pot oxidation-hydrolysis system
}

Suryani Saallah ${ }^{1 *}$, Jumardi Roslan ${ }^{2}$, Nurul Nadjwa Zakaria ${ }^{2}$, Wolyna Pindi², Shafiquzzaman Siddiquee ${ }^{1}$, Mailin Misson $^{1}$, Clarence M. Ongkudon ${ }^{1}$, Nur Hidayah Azmirah Mohd Jamil ${ }^{1}$, Wuled Lenggoro ${ }^{3}$

${ }^{1}$ Bioetchnology Research Institute, Universiti Malaysia Sabah, Jalan UMS, 88400 Kota Kinabalu, Sabah.

${ }^{2}$ Faculty of Food Science and Nutrition, Universiti Malaysia Sabah, Jalan UMS, 88400 Kota Kinabalu, Sabah.

${ }^{3}$ Institute of Engineering, Tokyo University of Agriculture and Technology, 2-24-16 Nakacho, Koganei, Tokyo, 184-8588, Japan.

**Corresponding author: Suryani Saallah, Bioetchnology Research Institute, Universiti Malaysia Sabah, Jalan UMS, 88400 Kota Kinabalu, Sabah, suryani@ums.edu.my

Abstract: In the present study, a facile one-pot production of nanocellulose from ripe and unripe Saba' banana (Musa acuminata $x$ balbisiana) peel was conducted by utilising hydrogen peroxide $\left(\mathrm{H}_{2} \mathrm{O}_{2}\right)$ as an oxidising agent prior to hydrolysis with sulphuric acid $\left(\mathrm{H}_{2} \mathrm{SO}_{4}\right)$ at different concentrations $(8 \%, 24 \%$ and $40 \%)$. Proximate and chemical compositions of the ripe and unripe banana peel (BP) powder were analysed, followed by physicochemical characterisations of the resulting nanocellulose by using Scanning Electron Microscopy (SEM), Fourier Transform Infrared (FTIR) spectroscopy and Dynamic Light Scattering (DLS). FTIR analysis has confirmed the successful removal of non-cellulosic components from the BP through the distinguishable spectra of both the ripe and unripe BP powder with the $\mathrm{H}_{2} \mathrm{O}_{2} / \mathrm{H}_{2} \mathrm{SO}_{4}$ - treated samples. SEM analysis revealed morphological changes of the BP powder from an irregular structure with the presence of starch granules to lamellar and fibrous structures after the $\mathrm{H}_{2} \mathrm{O}_{2} / \mathrm{H}_{2} \mathrm{SO}_{4}$ treatment and freeze-drying. The size of the nanocellulose is strongly influenced by the concentration of $\mathrm{H}_{2} \mathrm{SO}_{4}$ used. Nanocellulose from ripe BP produced by using the $40 \% \mathrm{H}_{2} \mathrm{SO}_{4}$ has the smallest size with $\mathrm{D} 50<80 \mathrm{~nm}$. These findings suggest the potential of banana peel, an abundant agricultural waste to be valorised into value-added materials with significant economic potentials.

Keywords: banana peel; nanocellulose; one-pot process; hydrogen peroxide pre-treatment; acid hydrolysis

Received: $18^{\text {th }}$ May 2020

Accepted: $11^{\text {th }}$ June 2020

Citation: Saallah S, Roslan J, Zakaria NN, et al. Isolation of nanocellulose from Saba' (Musa acuminata x balbisiana) banana peel by one-pot oxidation-hydrolysis system. Adv

Published: $26^{\text {th }}$ June 2020 


\section{Introduction}

The gradual depletion of petroleum sources has made it necessary for the investigation of renewable alternatives as a source of materials and energy. Therefore, research and development in nanocellulose production, a green, bio-based and renewable biopolymer is currently a subject of immense interest as a promising alternative to the conventional petroleum-based materials. A recent report by the Global Market Insights, Inc. (2019) forecasted that the global nanocellulose market will exceed USD 1 billion in 2024 with an impressive CAGR of $33.8 \%$. Owing to its desirable attributes such as eco-friendly nature, good biocompatibility, exceptional mechanical strength, tailorable surface chemistry and unique rheological behaviour, nanocellulose is viewed as an important advanced biomaterial that opens a new horizon in material science and its application. This includes as a filler or reinforced agent for composites and polymer matrixes, stabilising and thickening agents in food and cosmeceutical products, drug delivery excipient, biodegradable packaging, environmental remediation, among others (Camacho et al., 2017; Tibolla et al., 2018).

In the last few years, agricultural wastes have been utilised as an attractive source for nanocellulose production because these wastes consist mainly of plant fibres that are rich in cellulose, the main component of plant cell walls. Isolation and characterisation of nanocellulose from agricultural wastes such as sugarcane and cassava bagasse, pineapple fibres, vegetable and fruit peels such as potato, carrot, tomato and banana have been reported in a number of research papers (Malladi et al., 2018; Camacho et al., 2017; Moreno et al., 2018).

Banana is one of the world's most popular fruits and its cultivation is widespread in most tropical countries including Malaysia. Due to the high consumption and industrial processing of the edible parts of the banana, a huge quantity of by-products such as peel is generated. The peel which accounts for about $35 \%$ of the whole fruit weight is cellulose-rich residues. Orozco et al. (2014) reported that the cellulose content in banana peel (11.45\%) was higher than mango peel $(9.19 \%)$ and comparable to orange peel $(11.93 \%)$. Thus, this is a potential material for further utilisation (Tibolla et al., 2014; Harini et al., 2018). In this sense, research for the utilisation of banana peel as a source of nanocellulose has become increasingly attractive.

In the existing method of isolation of nanocellulose, pre-treatment followed by acid hydrolysis are the typical procedures by which the non-cellulosic components such as lignin and hemicellulose are deconstructed into soluble ones, leaving behind the insoluble residue of nanocellulose (Tibolla et al., 2017; Harini et al., 2018). However, the conventional methods usually involve several pre-treatment steps such as alkalisation, bleaching and successive washing to prepare relatively pure cellulosic starting materials prior to hydrolysis. These tedious and time-consuming steps will impede large-scale production and applications of nanocellulose (Leung et al., 2011). Moreover, the use of high acid concentration (typically in the range of 60 to $65 \%$ ) during hydrolysis possesses several downsides such as corrosive, high tendency of cellulose over-degradation, low yield, requires a huge amount of water for neutralisation and generates a huge amount of waste which eventually causes environmental pollution (Chen, 2017; Harini et al., 2018; Chen et al., 2019).

In view of these drawbacks, the present study reported for the first time a facile one-pot production of nanocellulose from ripe and unripe Saba' banana (Musa acuminata $x$ balbisiana) peel by utilising hydrogen peroxide as a bleaching agent prior to mild sulphuric acid hydrolysis with the concentration of $8 \%, 24 \%$ and $40 \%$, much lower than the commonly used acid concentration. Hydrogen peroxide $\left(\mathrm{H}_{2} \mathrm{O}_{2}\right)$ provides a safer alternative to the 
chemicals commonly used for alkalisation and bleaching of banana peel such as potassium hydroxide $(\mathrm{KOH})$, sodium hydroxide $(\mathrm{NaOH})$ and sodium chlorite $\left(\mathrm{NaClO}_{2}\right)$ because the decomposition of $\mathrm{H}_{2} \mathrm{O}_{2}$ produces water and oxygen thus, creating no harmful by-product, eliminating the needs of successive washing procedures and ultimately enabling the application of the one-pot process in less severe conditions (Chen et al., 2019). The findings of this study provide an insight into the improvement of the traditional nanocellulose isolation method from banana peel. Moreover, the utilisation of banana peel, an abundant agricultural waste would help to overcome environmental pollution issues and increase the economic value of the underutilised resources.

\section{Materials and Methods}

\subsection{Materials}

Unripe and ripe Saba' bananas (Musa acuminata $x$ balbisiana) with maturity stage 1 (green) and stage 7 (yellow peel with little brown spot), respectively, were obtained from a local market in Kota Kinabalu, Sabah, Malaysia. All the chemicals used in this study such as hydrogen peroxide and sulphuric acid are of reagent grade and were purchased from Merck Malaysia. A cellulose standard (microcrystalline) was purchased from Sigma-Aldrich. Millipore deionised water was used throughout this work.

\subsection{Preparation of Banana Peel (BP) Powder}

The BPs were manually removed from the flesh and immediately soaked in $2 \%$ sodium metabisulfite solution for $24 \mathrm{~h}$ to prevent oxidation. Next, the peels were arranged in an aluminium tray followed by drying in an oven at $60^{\circ} \mathrm{C}$ for $48 \mathrm{~h}$. The dried peels were then ground into fine powder and sieved through a 200-mesh sieve. The ripe and unripe BP powder was then stored in an airtight container at room temperature until further use. Procedures for the preparation of BP powder are as shown in Figure 1.

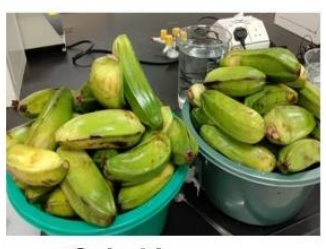

Saba' banana

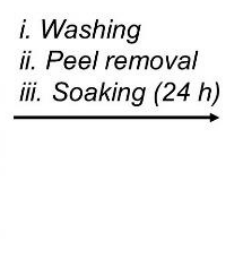

ii. Peel removal iii. Soaking $(24 h)$

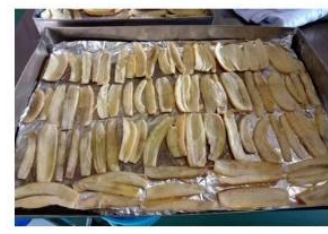

Banana peel (BP)

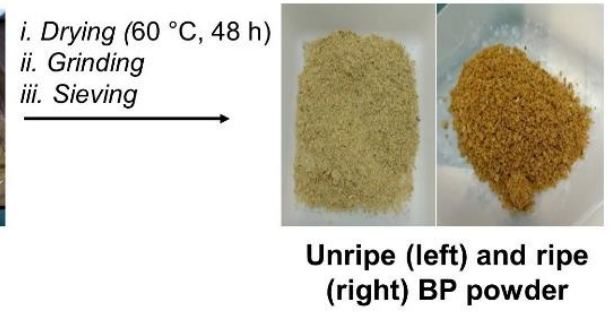

(right) BP powder

Figure 1. Procedures for the preparation of BP powder

\subsection{Isolation of BP Nanocellulose by One-Pot Process}

Fabrication of nanocellulose from the ripe and unripe BP powder was carried out by using a one-pot process following a method described by Chen et al. (2019) and Tibolla et 
al. (2018) with modifications. Briefly, $10 \mathrm{~g}$ of the dried BP powder was soaked in deionised water under continuous stirring for $24 \mathrm{~h}$ and filtered to remove water from the sample. After that, bleaching was performed with the addition of $100 \mathrm{ml}$ of $30 \mathrm{wt} \%$ hydrogen peroxide $\left(\mathrm{H}_{2} \mathrm{O}_{2}\right)$ to the swollen banana peel in a conical flask. The process was conducted at $90^{\circ} \mathrm{C}$ for $5 \mathrm{~h}$ with mechanical agitation in a shaking water bath (SW22, JULABO GmBH, Germany) to maintain the temperature. After completion of the bleaching process, the reaction mixture was cooled down to room temperature in an ice bath. Hydrolysis of the bleached suspension was performed by adding $8 \%, 24 \%$ and $40 \%$ of sulphuric acid $\left(\mathrm{H}_{2} \mathrm{SO}_{4}\right)$ into the same pot containing the suspension and heated to $80^{\circ} \mathrm{C}$ for $1 \mathrm{~h}$. To stop the reaction, the mixture was cooled with ice cubes. Successive washing with deionised water and centrifugation at 12000 rpm $\left(25^{\circ} \mathrm{C}\right)$ were conducted until constant $\mathrm{pH}$ was obtained to remove the non-fibrillated cellulose components and excess reactants and concentrate the nanocellulose. The final insoluble residue was then ultrasonicated in a pulsing mode for $5 \mathrm{~min}$ to improve the dispersibility and reduce agglomeration. Finally, the resulting suspension was lyophilised and stored in a sealed container at $4^{\circ} \mathrm{C}$ until further use. Figure 2 summarises the procedures for the isolation of nanocellulose from BP powder.
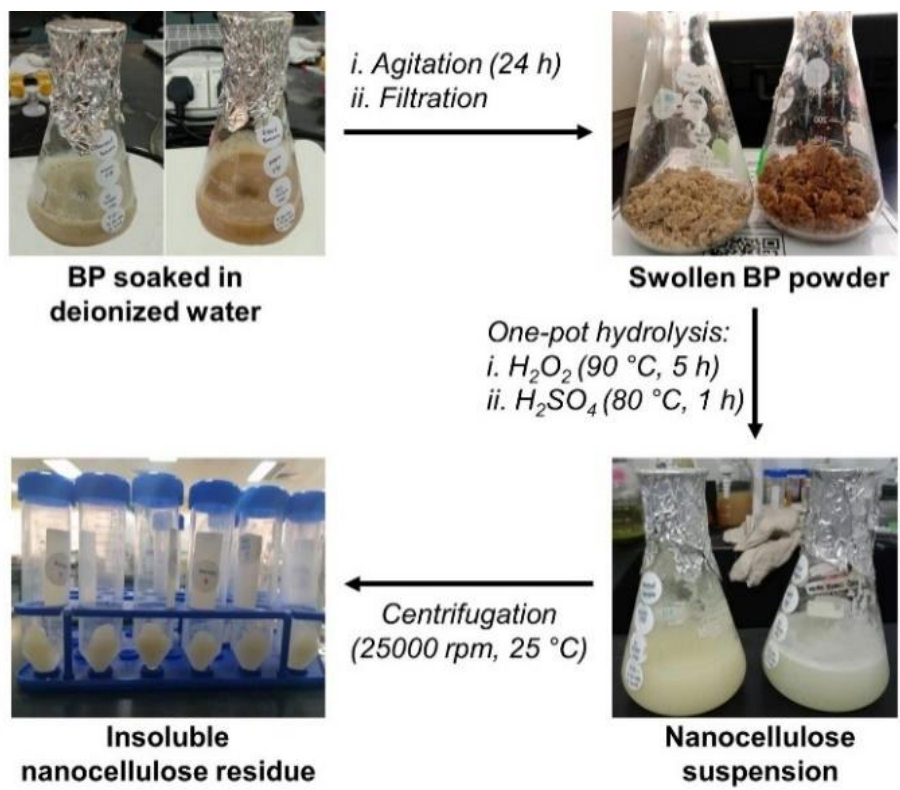

Figure 2. Steps involved in the isolation of nanocellulose from BP powder

\subsection{Characterisations of the BP Powder and Nanocellulose}

\subsubsection{Proximate compositions analysis}

Proximate compositions of the BP powder including moisture, crude protein, fat and ash contents were determined following the Association of Official Analytical Chemists (AOAC, $2005)$ procedure. Moisture content was determined by using the oven-dry method at $105^{\circ} \mathrm{C}$ 
for $3 \mathrm{~h}$ until it reached a constant weight. The Kjeldahl method was used to determine the crude protein content using Tecator Kjeltec protein analyser (FOSS, Hillerod, Denmark). The fat content was analysed using the Soxhlet method, while the ash content was measured using a muffle furnace ashing method in which the samples were heated in a furnace at $550^{\circ} \mathrm{C}$ for 8-12 h. All the analyses were performed in triplicates and the results were reported as average.

\subsubsection{Chemical compositions analysis}

Lignocellulosic fractions of the BP powder including cellulose, hemicellulose and lignin were analysed according to the American Society for Testing and Materials (ASTM) standard methods, ASTM D1103-60, ASTM D 1104-56 and ASTM D1106-56, respectively. Each test was conducted in duplicates, and the average values were reported.

\subsubsection{Fourier-Transform Infrared Spectroscopy (FTIR) Analysis}

Fourier-Transform Infrared Spectroscopy (FTIR) was used to identify functional groups present in the samples and changes in chemical compositions of the BP powder after hydrolysis with $8 \%, 24 \%$ and $40 \% \mathrm{H}_{2} \mathrm{SO}_{4}$. The absorbance spectra were collected using Agilent Cary 630 FTIR Spectrometer (Agilent Technologies Inc., USA) in the infrared region between 4000 and $600 \mathrm{~cm}^{-1}$ with a spectral resolution of $4 \mathrm{~cm}^{-1}$ and 32 scans at room temperature (Tibolla et al., 2018). FTIR spectrum of standard cellulose was used as a reference.

\subsubsection{Scanning Electron Microscope (SEM) Analysis}

Morphology of the ripe and unripe BP powder and the lyophilised nanocellulose suspensions were viewed by using SEM. The samples were fixed on an aluminium stub and sputter-coated with gold prior to the SEM observation. The analysis was performed using an accelerating voltage of $10 \mathrm{kV}$. Morphology of the standard cellulose was also observed for comparison.

\subsubsection{Dynamic Light Scattering (DLS) Analysis}

Particle size distribution of the nanocellulose suspensions was measured using NanoPlus (Particulate Systems, USA) particle analyser. The nanocellulose suspensions were prepared by diluting the lyophilised samples in ethanol $(0.1 \mathrm{wt} \%)$ followed by $5 \mathrm{~min}$ ultrasonication to improve sample dispersibility and reduce agglomeration. The DLS measurements were performed at room temperature $\left(25^{\circ} \mathrm{C}\right)$. The result is presented as the average value of three measurements.

\subsection{Statistical analysis}

The SPSS 25.0 was used to perform the statistical analysis of ANOVA and Tukey test of multiple comparisons. A $p$-value of less than 0.05 was considered significant. 


\section{Results and Discussion}

\subsection{Proximate and Chemical Compositions of the BP Powder}

Table 1 shows the proximate compositions of unripe and ripe BP powder. Both samples have relatively low moisture content $(7.13 \pm 0.12 \%$ and $7.26 \pm 0.02 \%$, respectively), indicating that the $\mathrm{BP}$ was less susceptible to microbial degradation and chemical changes, thus can be stored for a more extended period. As opposed to the unripe BP, the ripe BP has significantly higher ash, fat and protein contents $(\mathrm{p}<0.05)$, which agrees with the findings reported by Ramli et al. (2010) and Emaga et al. (2007). The ash content of the sample gives information about the mineral element composition, associated with the absorption of mineral salts by the plant. The higher fat content of the ripe BP could be due to the continuous synthesis of fatty acids during fruit metabolism. The slightly higher crude protein content of the ripe $\mathrm{BP}(7.48 \pm 0.36 \%)$ than that of the unripe $\mathrm{BP}(6.56 \pm 0.06 \%)$ can be explained by the breakdown and synthesis of the protein that occurs during fruit ripening. An increase in the crude protein content of BP with increasing maturity has also been reported by Khawas and Deka (2016).

Table 1. Proximate compositions of unripe and ripe BP powder*

\begin{tabular}{lcc}
\hline Component & Unripe BP powder & Ripe BP powder \\
\hline Moisture (\%) & $7.13 \pm 0.12$ & $7.26 \pm 0.02$ \\
Ash $(\%)$ & $10.16 \pm 0.03$ & $15.62 \pm 0.06$ \\
Fat $(\%)$ & $9.78 \pm 0.16$ & $13.59 \pm 0.11$ \\
Crude protein $(\%)$ & $6.56 \pm 0.06$ & $7.48 \pm 0.36$ \\
\hline
\end{tabular}

*Data are mean values of three replicates \pm standard deviation

Results of ASTM chemical compositions analysis of the ripe and unripe BP powder are shown in Table 2. Both samples have a considerable amount of cellulose that can be potentially exploited to produce nanocellulose. Based on the existing information on nanocellulose isolation from BP, most studies utilise the unripe BP as a source of cellulose instead of the ripe BP. But the present study found that the cellulose composition in the ripe BP powder is more than $50 \%$ higher than the unripe BP, associated with the increase in enzymatic activity of cellulose synthase during fruit ripening. This enzyme is responsible for the synthesis of cellulose in the primary and secondary walls of plant cells (Emaga et al., 2008). This finding is supported by Khawas and Deka (2016) where the cellulose content of plantain banana peel (Musa ABB) is different at ripening stages. 
As opposed to the cellulose content, the unripe BP shows significantly higher hemicellulose content than that of the ripe BP. Hemicellulose plays a vital role in maintaining the cell wall integrity together with lignin and cellulose. Cell biosynthesis, proliferation and prolongation at the early stages of fruit development are said to be the factors that contribute to the higher hemicellulose content of the unripe BP. Cell growth is modulated by enzymes that alter the structure of pectin and hemicellulose, thereby altering the interaction of these components. When growth is stopped, it is co-regulated by a reduction in the expression of the gene resulting in cell wall loosening and changes in the polysaccharide matrix leading to less cell extensible wall (Cosgrove, 2014). Lignification of the cell wall components during fruit ripening has resulted in higher lignin content in the ripe BP.

Table 2. Chemical compositions of unripe and ripe BP powder*

\begin{tabular}{lcc}
\hline Component & Unripe BP powder & Ripe BP powder \\
\hline Cellulose (\%) & $7.45 \pm 1.92$ & $11.89 \pm 1.02$ \\
Hemicellulose (\%) & $21.56 \pm 0.11$ & $15.26 \pm 0.67$ \\
Lignin (\%) & $7.31 \pm 1.14$ & $9.29 \pm 1.30$ \\
\hline
\end{tabular}

*Data are mean values of three replicates \pm standard deviation

\subsection{FTIR analysis}

FTIR spectroscopy analysis was used to identify changes in the chemical composition of the ripe and unripe BP in response to hydrolysis with different acid concentrations. As shown in Figure 3, the FTIR spectra of all samples have two major absorbance regions located in the high (3700-2800 $\left.\mathrm{cm}^{-1}\right)$ and low (1800-800 $\left.\mathrm{cm}^{-1}\right)$ wavenumbers. The broad -OH stretching vibration band in the $3700-3000 \mathrm{~cm}^{-1}$ region reflected the hydrophilicity of the banana fibre and gave essential information regarding inter- and intra-molecular hydrogen bond vibrations. The similarity of the absorption peak between the raw materials (unripe and ripe $\mathrm{BP}$ powder) to that of the treated samples at this region is a clear indication that the crystalline cellulose in the raw materials was not disrupted during the nanocellulose isolation process. The peak located at $\sim 2900 \mathrm{~cm}^{-1}$ was due to the $\mathrm{C}-\mathrm{H}$ stretching vibrations on polysaccharides (Chen et al., 2017).

Overall, the spectra of ripe and unripe BP powder are almost similar except for the appearance of a peak at $1075 \mathrm{~cm}^{-1}$ (pointed by the arrow in Figure 3(b)) for the unripe BP related to the presence of xylans associated with hemicellulose, which agrees well with the result of chemical composition analysis in Table 2 in which the unripe BP has higher hemicellulose content. The shoulder at $1730 \mathrm{~cm}^{-1}$ in the ripe and unripe BP powder was attributed to vibrations of the acetyl and uronic ester groups of hemicelluloses or to the ester linkage of the carboxylic group of the ferulic and p-coumaric acids of lignin (Mukwaya et al., 2017; Heloisa Tibolla et al., 2017). The gradual disappearance of this peak for the 
$\mathrm{H}_{2} \mathrm{O}_{2} / \mathrm{H}_{2} \mathrm{SO}_{4}$-treated samples indicated deacetylation of hemicellulose and delignification. An obvious peak at $1640 \mathrm{~cm}^{-1}$ for the unripe BP powder is usually associated with adsorbed water and could also originate from carbonyl stretching conjugated with aromatic rings as observed by Tibolla et al. (2014) for nanocellulose isolated from Musa paradisiaca banana peel (Heloisa Tibolla et al., 2014). For the ripe BP, the peak is located at lower wavenumber region $\left(1603 \mathrm{~cm}^{-1}\right)$ associated with aromatic skeletal vibration (Han et al., 2013). These peaks were gradually diminished as the ripe and unripe BP powder underwent one-pot hydrolysis with increasing acid concentration, indicating the removal of most of the lignin and hemicellulose fractions during the nanocellulose isolation process. The peak at $897 \mathrm{~cm}^{-1}$ which referred to typical cellulose structure (Chen et al., 2019) is not observed in the untreated samples but appears in the spectra of the standard and all the $\mathrm{H}_{2} \mathrm{O}_{2} / \mathrm{H}_{2} \mathrm{SO}_{4}$-treated samples confirming successful isolation of cellulose and nanocellulose from the BP powder.

(a)

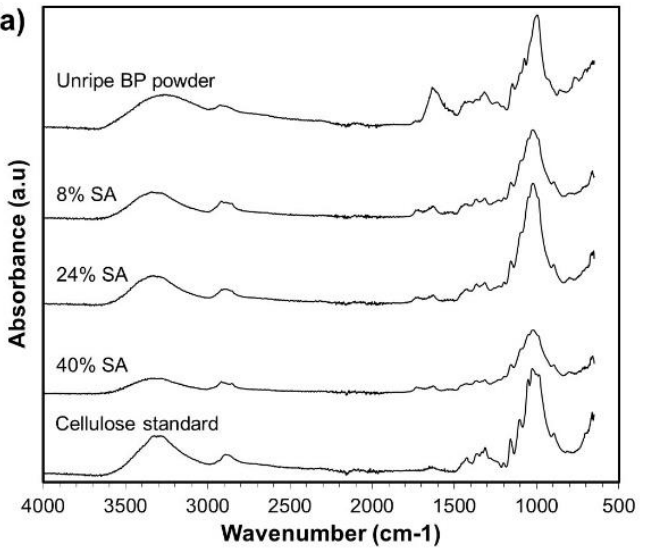

(c)

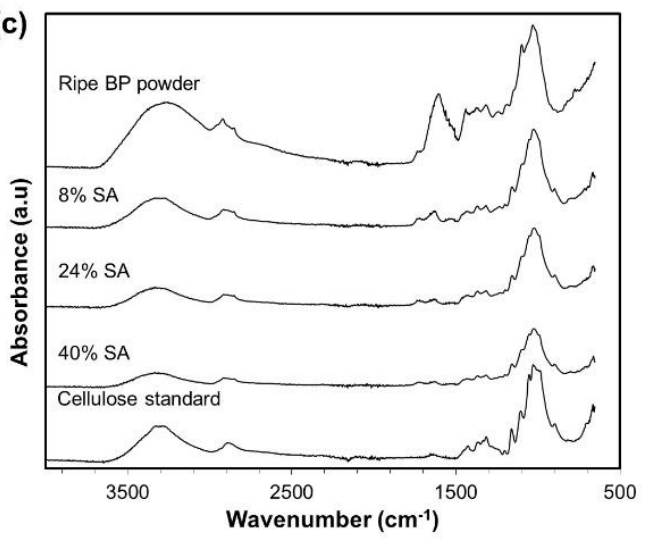

(b)

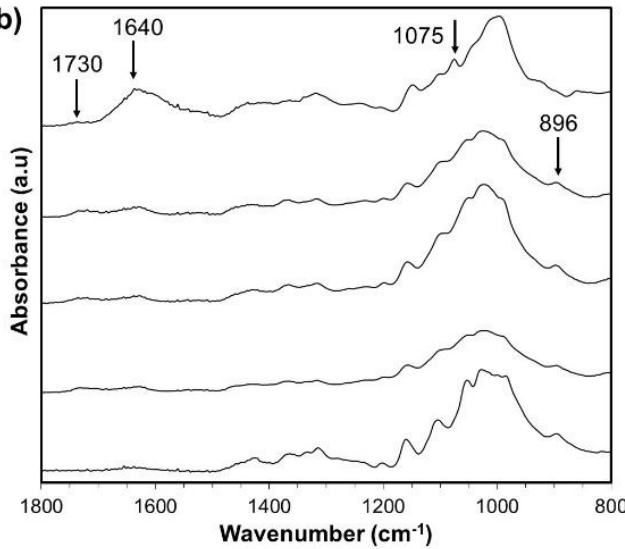

(d)

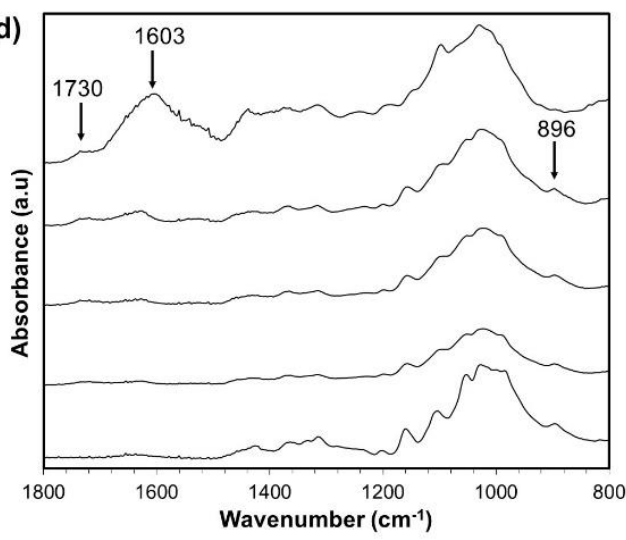

Figure 3. FTIR spectra of (a, b) unripe BP samples and (c, d) ripe BP samples after $\mathrm{H}_{2} \mathrm{O}_{2} / \mathrm{H}_{2} \mathrm{SO}_{4}$ treatment with different acid concentrations. Spectra of untreated ripe and BP powder and cellulose standard were also plotted for comparison. 


\subsection{Morphological characterisation}

After the one-pot isolation process, the resulting nanocellulose suspensions were freezedried and their morphological structure was characterised by SEM as presented in Figure 4. The standard cellulose exhibited a compact structure (Figure 4a) which was attributed to the strong and tightly packed hydrogen bonding networks of cellulose fibrils which made them highly ordered and rigid. The unripe BP powder shows an irregular structure with rough surfaces (Figure 4b), while the ripe BP has a more consistent globular structure with the presence of starch granules (Figure 4c). Different morphology of the ripe and unripe BP powder could be due to the variation in proximate and chemical compositions.
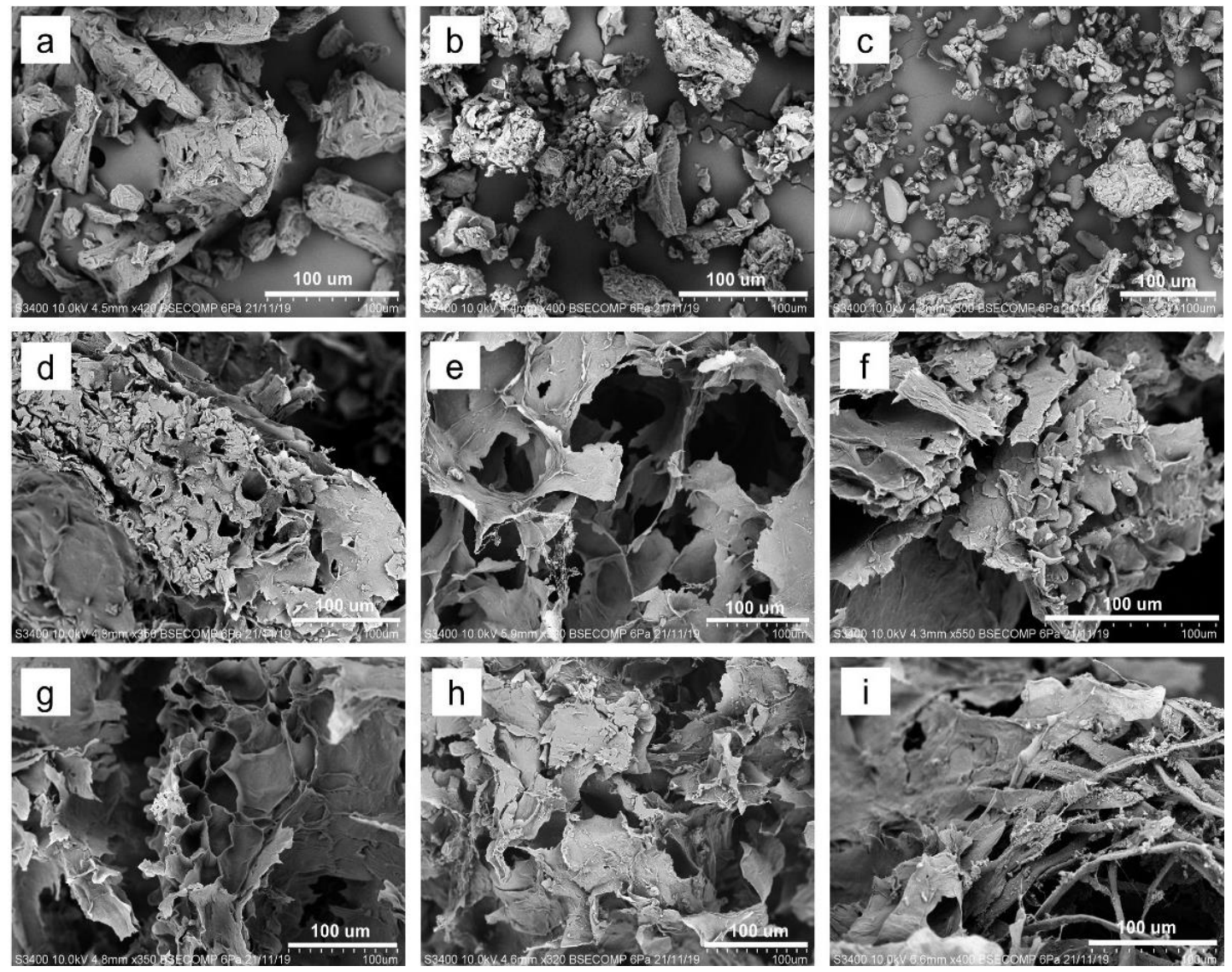

Figure 4. SEM images of (a) microcrystalline cellulose (standard), (b) unripe BP powder, (c) ripe BP powder, (d-f) $\mathrm{H}_{2} \mathrm{O}_{2} / \mathrm{H}_{2} \mathrm{SO}_{4}$-treated unripe $\mathrm{BP}$ after $\mathrm{H}_{2} \mathrm{O}_{2} / \mathrm{H}_{2} \mathrm{SO}_{4}$ treatment with $8 \%, 24 \%$ and $40 \%$ sulphuric acid, respectively and (g-i) ripe BP after $\mathrm{H}_{2} \mathrm{O}_{2} / \mathrm{H}_{2} \mathrm{SO}_{4}$ treatment with $8 \%, 24 \%$ and $40 \%$ sulphuric acid, respectively.

Regardless of the BP source (ripe or unripe) and acid concentration, all the isolated nanocellulose samples appear as a porous network with a unique lamellar structure (Figure $4 \mathrm{~d}$ to $4 \mathrm{i}$ ). Closer observation revealed ultrafine fibres and particles adhere to the nanocellulose surface. Interestingly, a honeycomb structure can be clearly seen in Figure 4 (g). This structure is related to the good stability of the nanocellulose in colloidal suspension (Lavoine \& Bergström, 2017). 
Possible mechanism of the formation of lamellar structure upon freeze-drying of nanocellulose could be inferred from the strong hydrogen bonding between the cellulose subunits, promoting self-assembly of the nanocellulose (Han et al., 2013). Before freezing, the nanocellulose was initially stable due to electrostatic repulsion between the negatively charged sulphate groups present on its surface. As the freeze-drying progresses, the development of ice crystals gradually forms a lamellar microstructure. This process causes the nanocellulose particles to concentrate and be trapped in the space between these ice crystals. As a result, the nanocellulose was reorganised in a longitudinal direction and induced self-assembly followed by the formation of larger cellulose clusters via hydrogen and van der Waals bonds.

Han et al. (2013) also postulated that the self-assembly mechanism of nanocellulose suspension is predominantly influenced by the concentration of nanocellulose in the suspension. Generally, at higher acid concentration, a higher concentration of nanocellulose suspension was produced. In this case, the gap between the self-assembled microfibre layers was very small, thus increasing the tendency of forming hydrogen bonding with the adjacent microfibres. These microfibres were aligned to form a dense, layered structure, as observed in Figure 4(f) and 4(i) for nanocellulose isolated with $40 \%$ acid concentration. In contrast, the nanocellulose that was isolated using lower acid concentration (8\% and $24 \%$ ) tended to form a loose structure with bigger space between the self-assembled microfibres owing to the weakening hydrogen bonding and interfacial attraction.

\subsection{Particle size distribution}

A dynamic light scattering (DLS) analysis was used to analyse the size of the nanocellulose in the suspension and the results are presented in a cumulative frequency distribution. As shown in Figure 5, the average hydrodynamic size of the standard cellulose is $42918.6 \pm 9658.2 \mathrm{~nm}$, consistent with the size given by the manufacturer, indicating the reliability of the results obtained from the DLS measurements. The unripe BP powder subjected to the one-pot isolation process with sulphuric acid of $8 \%, 24 \%$ and $40 \%$ concentrations yielded nanocellulose with average sizes of $362.2 \pm 191.5 \mathrm{~nm}, 328.1 \pm 140.8$ $\mathrm{nm}$ and $176.1 \pm 48.6 \mathrm{~nm}$, respectively (Figure $5 \mathrm{a}$ ). For the ripe $\mathrm{BP}$, the average sizes of the isolated nanocellulose are $701.6 \pm 226.1 \mathrm{~nm}, 258.6 \pm 111.2 \mathrm{~nm}$ and $78.6 \pm 72.0 \mathrm{~nm}$, respectively (Figure 5b). These results indicate that the source of raw material (ripe or unripe) and concentration of acid used for hydrolysis have a significant influence on the size of the isolated nanocellulose. The shown hydrodynamic size appears to decrease as the acid concentration increases because, during acid hydrolysis, the $\beta-1,4$ glycoside bond of cellulose is broken which causes the polymer chain to shorten and thus decreases the physical dimension (Camacho et al., 2017).

Successful isolation of nanocellulose from BP powder can be confirmed as more than $95 \%$ of the volume fraction of the particles lies in the nanometric range, except for the ripe BP powder treated with $8 \%$ sulphuric acid. This could be due to the higher lignin content of 
the raw material as presented earlier in the chemical composition and FTIR analyses. The ripe $\mathrm{BP}$ powder underwent about 10 folds reduction in size with increasing acid concentration from $8 \%$ to $40 \%$ while for the unripe BP, only two folds size reduction was observed, respectively. From all the samples investigated, the ripe BP powder treated with $40 \%$ sulphuric acid exhibited the smallest hydrodynamic size in which about $70 \%$ of the particles are having a size below $100 \mathrm{~nm}$. However, the definite reason as to why the size reduction in ripe $\mathrm{BP}$ samples is more pronounced than the unripe $\mathrm{BP}$ with increasing acid concentration is unclear and rarely discussed. Moreover, to our knowledge, a comparison of nanocellulose isolation from ripe and unripe BP samples has never been reported. Therefore, future studies should focus on investigating the structural differences between the ripe and unripe BP powders that could elucidate these interesting findings.

(a) Unripe BP

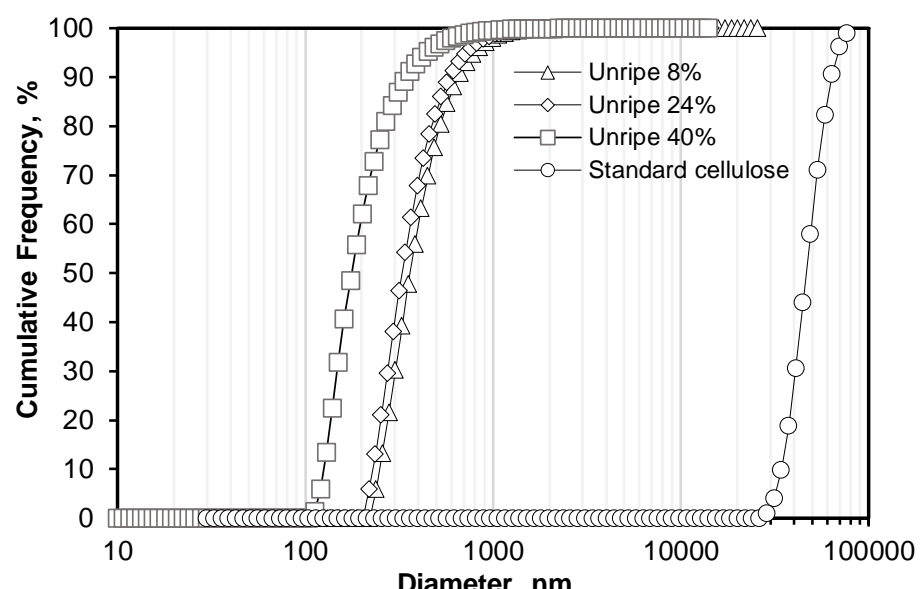

(b) Ripe BP

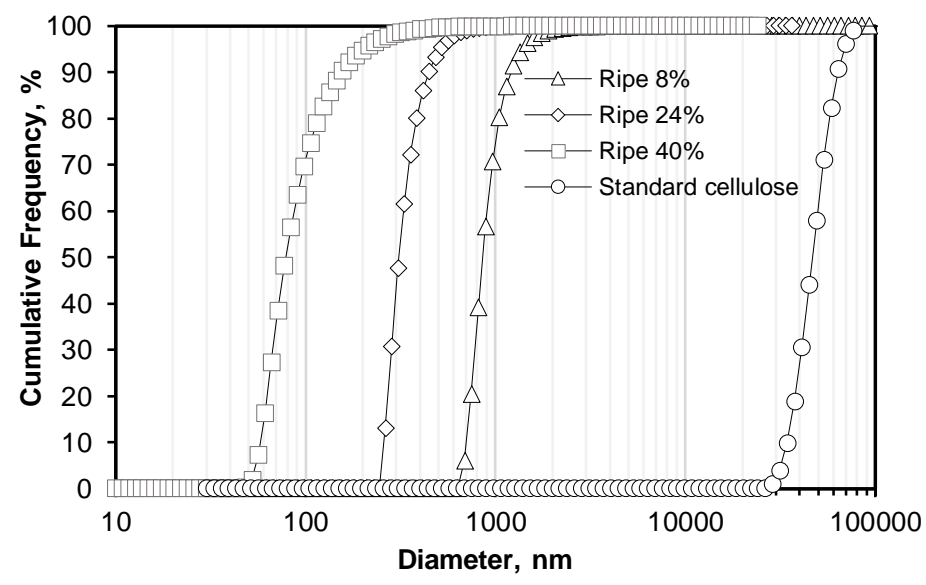

Figure 5. DLS analysis of ripe and unripe BP powder after one-pot $\mathrm{H}_{2} \mathrm{O}_{2} / \mathrm{H}_{2} \mathrm{SO}_{4}$ treatment with different acid concentration. Result of standard cellulose.

The use of $60-65 \% \mathrm{H}_{2} \mathrm{SO}_{4}$ has been extensively reported for isolation of nanocellulose from various sources such as sugar beet (Leitner et al., 2007), sugarcane bagasse (Mandal \& 
Chakrabarty, 2011) and pomelo peel (Mat Zain et al., 2014) with sizes of 30-100 nm, 18$220 \mathrm{~nm}$ and 100-150 nm, respectively. This is due to the premise that lower acid concentration would prevent the formation of nanocellulose (Camacho et al., 2017; Maciel et al., 2019; Shanmugarajah et al., 2015). However, in this study, it is proven that nanocellulose can be isolated from banana peel under milder acid hydrolysis conditions with the aid of hydrogen peroxide as a green oxidising agent.

\section{Conclusion}

The present study has discovered the potential of unripe and ripe Saba' banana peel, underutilised lignocellulosic biomass to be valorised into nanocellulose. The unripe and ripe $\mathrm{BP}$ are having different proximate and chemical compositions, which influence the properties of the resultant nanocellulose. The application of one-pot oxidation-hydrolysis approach by utilising hydrogen peroxide as a green oxidising agent prior to hydrolysis with sulphuric acid at mild concentrations is proven effective for the isolation of nanocellulose. The nanocellulose exhibits a porous lamellar structure, typical behaviour of nanocellulose under freeze-drying condition while the chemical structure resembles those of the standard cellulose as verified by FTIR. Besides the source of the material (ripe or unripe), the concentration of sulphuric acid used has a strong impact on the size of the nanocellulose. This observation has been validated by the DLS results by which higher concentration of sulphuric acid favoured solubilisation of the non-cellulosic components including lignin and hemicellulose and facilitate isolation of nanocellulose with smaller size. The size reduction in ripe BP is more pronounced than the unripe BP with increasing acid concentration. However, the reason behind this finding remains unclear and calls for further investigation. Overall, the one-pot approach employed in this study provides a simpler alternative to the laborious and time-consuming conventional approach for isolation of nanocellulose from banana peel.

Author Contributions: Conceptualization, S.S*., J.R., W.P., and W.L.; funding acquisition, S.S*., J.R., M.M., S.S., and C.M.O.; investigation, N.N.Z. and N.H.A.M.J.; writing—original draft preparation, N.N.Z. and J.R.; writing—review and editing, S.S*, M.M., and W.L.

Funding: This research was funded by the Ministry of Education, Malaysia under FRGS-RACER scheme (RACER/1/2019/TK10/UMS//1) and Universiti Malaysia Sabah (PHD0020-2019).

Conflicts of Interest: The authors declare no conflict of interest.

\section{References}

Camacho, M., Ureña, Y. R. C., Lopretti, M., et al. (2017). Synthesis and characterization of nanocrystalline cellulose derived from Pineapple peel residues. Journal of Renewable Materials, 5(3-4), 271-279. https://doi.org/10.7569/JRM.2017.634117

Chen, Y. W. (2017). Isolation and Characterization of Nanocrystalline Cellulose From Oil Palm Biomass Via Transition Metal Salt Catalyzed Hydrolysis Process. Retrieved from http://studentsrepo.um.edu.my/7710/6/Thesis_(Chen_You_Wei\%2C_HGA_140012).pdf 
and porous network based nanocellulose synthesis via one-pot hydrolysis system. International Journal of Biological Macromolecules, 123, 1305-1319. https://doi.org/10.1016/j.ijbiomac.2018.10.013

Chen, Y. W., Tan, T. H., Lee, H. V., et al. (2017). Self-assembling behavior of cellulose nanoparticles during freeze-drying: Effect of suspension concentration, particle size, crystal structure, and surface charge. Biomacromolecules, 14(5), 1529-1540. https://doi.org/10.1021/bm4001734

Emaga, H. T., Andrianaivo, R. H., Wathelet, B., et al. (2007). Effects of the stage of maturation and varieties on the chemical composition of banana and plantain peels. Food Chemistry, 103(2), 590-600.

Emaga, H. T., Robert, C., Ronkart, S. N., et al. (2008). Dietary fibre components and pectin chemical features of peels during ripening in banana and plantain varieties. Bioresource Technology, 99(10), 4346-4354. https://doi.org/10.1016/j.biortech.2007.08.030

Global Market Insights, Inc. (2019). Retrieved from https://www.gminsights.com/pressrelease/nanocellulose-market

Han, J., Zhou, C., Wu, Y., et al. (2013). Self-assembling behavior of cellulose nanoparticles during freeze-drying: Effect of suspension concentration, particle size, crystal structure, and surface charge. Biomacromolecules, 14(5), 1529-1540. https://doi.org/10.1021/bm4001734

Harini, K., Ramya, K., \& Sukumar, M. (2018). Extraction of nano cellulose fibers from the banana peel and bract for production of acetyl and lauroyl cellulose. Carbohydrate Polymers, 201(June), 329-339. https://doi.org/10.1016/j.carbpol.2018.08.081

Khawas, P., \& Deka, S. C. (2016). Comparative Nutritional, Functional, Morphological, and Diffractogram Study on Culinary Banana (Musa ABB) Peel at Various Stages of Development. International Journal of Food Properties, 19(12), 2832-2853.

Lavoine, N., \& Bergström, L. (2017). Nanocellulose-based foams and aerogels: Processing, properties, and applications. Journal of Materials Chemistry A, 5(31), 16105-16117. https://doi.org/10.1039/c7ta02807e

Leitner, J., Hinterstoisser, B., Wastyn, M., Keckes, J., \& Gindl, W. (2007). Sugar beet cellulose nanofibril-reinforced composites. Cellulose, 14(5), 419-425. https://doi.org/10.1007/s10570-007-9131-2

Leung, A. C. W., Hrapovic, S., Lam, E., Liu, Y., et al. (2011). Characteristics and properties of carboxylated cellulose nanocrystals prepared from a novel one-step procedure. Small, 7(3), 302-305. https://doi.org/10.1002/smll.201001715

Maciel, M. M. Á. D., Benini, K. C. C. de C., Voorwald, H. J. C., et al. (2019). Obtainment and characterization of nanocellulose from an unwoven industrial textile cotton waste: Effect of acid hydrolysis conditions. International Journal of Biological Macromolecules, 126, 496-506. https://doi.org/10.1016/j.ijbiomac.2018.12.202

Malladi, R., Nagalakshmaiah, M., Robert, M., et al. (2018). Importance of agriculture and industrial waste in the field of nano cellulose and its recent industrial developments: A review. ACS Sustainable Chemistry \& Engineering, 6(3), 2807-2828. https://doi.org/10.1021/acssuschemeng.7b03437

Mandal, A., \& Chakrabarty, D. (2011). Isolation of nanocellulose from waste sugarcane bagasse (SCB) and its characterization. Carbohydrate Polymers, 86(3), 1291-1299. https://doi.org/10.1016/j.carbpol.2011.06.030

Mat Zain, N. F. (2014). Preparation and Characterization of Cellulose and Nanocellulose From Pomelo (Citrus grandis) Albedo. Journal of Nutrition \& Food Sciences, 05(01), 10-13. https://doi.org/10.4172/2155-9600.1000334

Moreno, G., Ramirez, K., Esquivel, M., et al. (2018). Isolation and characterization of nanocellulose obtained from industrial crop waste resources by using mild acid hydrolysis. Journal of Renewable Materials, 6(4), 362-369. https://doi.org/10.7569/JRM.2017.634167 
Mukwaya, V., Yu, W., Asad, R. A. M., et al. (2017). An environmentally friendly method for the isolation of cellulose nano fibrils from banana rachis fibers. Textile Research Journal, 87(1), 81-90. https://doi.org/10.1177/0040517515622155

Orozco, R. S., Hernández, P. B., Morales, G. R., et al. (2014). Characterization of lignocellulosic fruit waste as an alternative feedstock for bioethanol production. BioResources, 9(2), 1873-1885.

Ramli, S., Ismail, N., Alkarkhi, A. F. M., et al. (2010). The use of principal component and cluster analysis to differentiate banana peel flours based on their starch and dietary fibre components. Tropical Life Sciences Research, 21(1), 91100.

Shanmugarajah, B., Kiew, P. L., Chew, I. M. L., et al. (2015). Isolation of Nanocrystalline Cellulose (NCC) from palm oil empty fruit bunch (EFB): Preliminary result on FTIR and DLS analysis. Chemical Engineering Transactions, 45(October), 1705-1710. https://doi.org/10.3303/CET1545285

Tibolla, H., Pelissari, F. M., Martins, J. T., et al. (2018). Cellulose nanofibers produced from banana peel by chemical and mechanical treatments: Characterization and cytotoxicity assessment. Food Hydrocolloids, 75, 192-201.

Tibolla, H, Pelissari, F. M., Martins, J. T., et al. (2018). Food Hydrocolloids Cellulose nano fibers produced from banana peel by chemical and mechanical treatments: Characterization and cytotoxicity assessment. Food Hydrocolloids, 75, 192-201. https://doi.org/10.1016/j.foodhyd.2017.08.027

Tibolla, H., Pelissari, F. M., \& Menegalli, F. C. (2014). Cellulose nanofibers produced from banana peel by chemical and enzymatic treatment. LWT - Food Science and Technology, 59(2P2), 1311-1318. https://doi.org/10.1016/j.lwt.2014.04.011

Tibolla, H., Pelissari, F. M., Rodrigues, M. I., et al. (2017). Cellulose nanofibers produced from banana peel by enzymatic treatment: Study of process conditions. Industrial Crops and Products, 95, 664-674. https://doi.org/10.1016/j.indcrop.2016.11.035 